\title{
Effect of Smartphone Addiction on Neck Function among Undergraduate Physical Therapist Students
}

Rania N. Karkusha*, Dalia M. Mosaad", Basant S. Abdel Kader**

* Basic Science Department, Faculty of Physical Therapy, Cairo University, ** Basic Science Department,

Faculty of Physical Therapy, Misr University for Science and Technology

Corresponding author: Basant S. Abdel Kader, Mobile: 01091445676, Email: pasantsaed@gmail.com

\begin{abstract}
Background: the prevalence of gadget was increased between university students. They use it for entertainment rather than learning. The long use of smartphone would be risk factor to musculoskeletal disorder, early neck pain, neck problems and poor work habits that load cervical region. Purpose: this study was conducted to study the effect of smartphone addiction on cervical range of motion and neck function among undergraduate students in the faculties of physical therapy.

Design: Cross-sectional one-shot, controlled observational study. Methods: one hundred students selected from different physical therapy colleges in Cairo and Giza using short version of smartphone addiction scale (SAS sv). For selection, the students were assigned into two groups; Group A, consisted of 62 students who were non-addicted smartphone users and Group B, consisted of 38 students who were addicted smartphone users. Cervical range of motion (ROM) assessed by CROM and Copenhagen neck function disability index was used to assess neck function.

Result: There were no statistical significant differences between both groups regarding BMI., regard to the cervical range of motion there were statistical significant differences between both group in all direction (flexion, left lateral flexion, right lateral flexion, left rotation and right rotation) except extension and Copenhagen neck functional disability index was significantly higher among smartphone addicted group. Conclusion: Smartphone addiction affects cervical range of motion and neck function so, it is recommended that physical therapy students should not use smartphone in addicted manner to prevent long term neck disability. Ergonomics advises for smartphone use will be preventive therapy programmers.
\end{abstract}

Keywords: smartphone addiction, neck function, undergraduate students in the college of physical therapy.

\section{INTRODUCTION}

In Egypt, the percentage of the number of mobile phone users increased from $75 \%$ in 2012 to $80 \%$ in 2013 on a sample of 1,000 users. More than $73 \%$ of the internet users are tertiary students, which include university students ${ }^{(\mathbf{1})}$, also a different national survey revealed that smartphone addiction is twice more prevalent among teenagers than among adults ${ }^{(2)}$. Nowadays, the use of visual display terminal (VDT) of computer and smartphones in almost all homes and organizations is very common ${ }^{(3)}$. , individuals utilize smartphones for different tasks on a daily basis, might lead to bad postures ${ }^{(4)}$.

Furthermore, the usage of smartphones was reported to cause significant alterations in upper cervical posture ${ }^{(\mathbf{5})}$. It is also known that misalignment of the upper cervical posture can induce alterations in muscular tone, musculoskeletal dysfunction. Other studies found that smartphone use would lead to muscular fatigue and text neck, which are the most common condition, as well as a decreased cervical range of motion ${ }^{\left({ }^{6}, 7\right)}$. Studies that tested physical exposure related to the use of smartphones are lacking today therefore, it is necessary to evaluate the physical changes that occur during the use of smartphones, and determine their use as a risk factor that could induce musculoskeletal system disorders, In a previous study the cervical mobility in the mobile addiction group was significantly decreased in lateral flexion, cervical flexion, and cervical extension ${ }^{\mathbf{8}}$, ${ }^{\mathbf{9}, 10)}$. The Young study ${ }^{(\mathbf{1 0})}$ concerned with effect of mobile phone addiction on mobility of cervical region during use of smartphone recommended that further study is needed to find the relation between smartphone addiction and limitation of ROM of cervical joints, further considerations are required to outline the negative impacts of addiction of smartphones on cervical mobility and to implement preventive measures ${ }^{(11)}$.

Aim of the study is to determine the effect of smartphone addiction on cervical range of motion and cervical functions among physical therapy college students.

\section{METHODS}

The present study was concerned with the effect prolonged use of smartphone on range of motion among undergraduate physical therapist. It was conducted in different faculties of physical therapy as Cairo University outpatient clinic, Egyptian Chines University, Egyptian Veterans and War Victims Association Physical Therapy Department, where different physical therapy universities students spent the training courses there (BADR University, MTI University and 6 October University).

\section{Design:}

Study design: Cross-sectional one-shot, controlled observational study design.

Study variable: (dependent variables: smartphone addiction, independent variable: range of motion and neck function)

Duration of data collection: from November 2018 to January 2019

Trial repetition: three repetitions for each CROM measurements 
Sample:

Sample size: one hundred undergraduate physical therapist

Demographic data: (age: $21.08 \pm 1.86$ years, weight: $64.82 \pm 8.19 \mathrm{~kg}$, height: $1.68 \pm 0.09 \mathrm{~cm}$ and body mass index $23.07 \pm 2.29 \mathrm{~kg} / \mathrm{m}^{2}$ )

Study groups: - Group (A): is (62 non-addicted smartphone users).

- Group (B): is (38 smartphone addicted users).

\section{Randomization technique:}

Short version of smartphone addiction scale (SAS sv) to explore the pattern of mobile phone usage these self reporting questionnaire distributed among undergraduate students as interview to examiner in the faculties of physical therapy ${ }^{(12,13)}$.

Ethical approval: The study was approved by the Ethics Board of Cairo University.

All subjects enrolled in the study assigned a written consent.

The participants were included if the age of participants ranged from $19-24$ years, both genders were included, RT handed subject. Person who use phone without hand free as hand held mobile texting on screen or watching media on relatively small screen for long period of time exceeding 4 hours per day, person uses the smartphones for not less than three years, person uses same smartphone for not less than one year. Size of mobile screen should be not less than 4 inches measured by the diagonal line at RT corner down to its corner. ${ }^{(2,17,23)}$

The participants were excluded if they had any head and neck injuries or surgeries within pervious one year before the study, any congenital abnormalities or symptomatic deformity in either the cervical or the lumbar spine, any uncorrected visual or auditory problems, dizziness, vertigo and headache that may affect the subject to participate at the tests, any systematic diseases, any addiction to alcohol, any additive drugs and sedatives within 48 hours previous to tests, body mass index of subjects over $25 \mathrm{~kg} / \mathrm{m}^{2(6,7)}$.

\section{Instrumentations}

Smartphone addiction scale short version (SAS sv)

Smartphone addiction scale short version (SAS sv) was used for this age group. It is a reliable and valid assessment tool that was originally developed by (Kwon et al, 2103) ${ }^{(\mathbf{1 3 )}}$ and revised to a short form for adolescents containing 10 items. The scale is coded from $1=$ "fully disagree" to $6=$ "fully agree."

It is used to a short-version scale in order to evaluate the grades of addiction.

Copenhagen Neck Functional Disability Scale (CNFDS) The CNFDS clinical evaluation tool that accurately reflects the patient's perception regarding his/her functionality with it, as fifteen items that assess the impact of neck pain and disability level. Three items assess the pain severity, including the patient's acuity of the future impact of neck pain; eight items assess the disability during every-day activities; and four items focus on social interaction and recreation ${ }^{(\mathbf{1 2})}$.

Afterwards, we add up all the scores of the questions to form the total score ranges which ranges from 0 to 30 . The total score determines the level of functional disability, in which higher numbers represent a higher level of disability ${ }^{(14)}$.

\section{Procedure}

\section{I- Anthropometric measures:}

- $\quad$ Body weight and height was measured

- $\quad$ Body mass index was calculated

\section{II- Subject's preparation}

Cervical active ROM test measured for each participant in the study by CROM device as follow: From sitting position, the participant's feet were placed flat on the floor and arm supported on the students lap Knees and elbow joint angles were at $90^{\circ}$. The chair did not extend above the suprascapular border, and hence did not restrict head or neck motion. Each subject was asked to move his head as far as possible in each of the 4 directions. Cervical Flexion, extension, right lateral bending, left lateral bending; rotation right and left rotation were measured. For higher reliability, all measurements were performed 3 times. The mean value of 3 consecutive measurements was calculated and used for data analysis (15).

The researcher instructed the subjects orally not to move their trunk and to keep the posture .

For measuring different cervical angles:

- For flexion 0-60 $60^{\circ}$

- For extension $0-50^{\circ}$,

- For lateral flexion $0-50^{\circ}$,

- For rotation $0-55^{\circ}$.

Statistical analysis of data:

It was carried out using the SPSS computer package version 25.0 (IBM SPSS Statistics for Windows,

Armonk, NY: IBM Corp., USA).

- Normality was checked for study variable by

Shapiro test and revealed no significant differences

for all dependent variable.

- Independent samples t-test was applied.

- Multivariate Analysis of Variance (MANOVA) was carried out to compare the mean values of dependent variables between both groups using Wilks' Lambda effect.

- The statistical methods were verified, assuming a significant level of $\mathrm{p}<0.05$.

\section{RESULTS}

The demographic data of the two groups, smartphone addicted group $(n=38)$ and non-addicted group $(n=62)$ are represented in table 1 with their level of addiction according to SAS sv and the mean of smartphone addiction scale was significantly higher among smartphone addicted group $(\mathrm{P}<0.001)$. 
Table (1): General characteristics and anthropometry measurements of the studied groups.

\begin{tabular}{|l|c|c|c|}
\hline \multirow{2}{*}{ Variables } & $\begin{array}{c}\text { Non-addicted group (A) } \\
(\mathbf{n = 6 2})\end{array}$ & $\begin{array}{c}\text { Addicted group (B) } \\
(\mathbf{n}=\mathbf{3 8})\end{array}$ & \multirow{2}{*}{ P- value } \\
\cline { 2 - 3 } & Mean $\mathbf{\text { SD }}$ & Mean \pm SD & \\
\hline Age (years) & $21.39 \pm 1.64$ & $21.08 \pm 1.86$ & $>0.05$ \\
\hline Weight $($ Kg) & $62.02 \pm 8.11$ & $64.82 \pm 8.19$ & $>0.05$ \\
\hline Height (m) & $1.65 \pm 0.06$ & $1.68 \pm 0.09$ & $>0.05$ \\
\hline BMI & $23.12 \pm 2.32$ & $23.07 \pm 2.29$ & $>0.05$ \\
\hline $\begin{array}{l}\text { SAS-SV } \\
\text { Mean } \pm \text { SD }\end{array}$ & $26.34 \pm 8.54$ & $46.42 \pm 5.26$ & $<0.001^{*}$ \\
\hline
\end{tabular}

Values present as mean \pm SD were analyzed by independent samples t-test. *: Significant.

Multivariate analysis of variance (MANOVA) was carried out to compare the mean values of CNFDI, ROM variables between non-addicted and smartphone addiction groups. Based on MANOVA calculations, there is a significant group effect with Wilks' Lambda $=0.58, \mathrm{~F}(13,86)=4.78, \mathrm{p}<0.001$; partial eta squared $=0.419$ (reflecting large effect size) These results indicate that there are significant differences in CNFDI, ROM variables between the two groups (non-addicted and smartphone addiction groups) as represented in table (2) and figure (1).

Table (2): Range motion and Copenhagen neck function disability index.

\begin{tabular}{|l|c|c|c|}
\hline \multicolumn{1}{|c|}{ Variables } & $\begin{array}{c}\text { Non-addicted group (A) } \\
(\mathbf{n = 6 2 )}\end{array}$ & $\begin{array}{c}\text { Addicted group (B) } \\
(\mathbf{n = 3 8 )}\end{array}$ & P-value \\
\hline Flexion & $60.35 \pm 12.29$ & $55.51 \pm 10.25$ & $0.044^{*}$ \\
\hline Extension & $37.72 \pm 6.1$ & $36.77 \pm 8.21$ & $>0.05$ \\
\hline Right lateral flexion & $45.04 \pm 7.93$ & $41.24 \pm 7.62$ & $0.020^{*}$ \\
\hline Left lateral flexion & $45.47 \pm 8.22$ & $41.23 \pm 6.37$ & $0.008^{*}$ \\
\hline Right rotation & $60.88 \pm 14.0$ & $55.66 \pm 8.16$ & $0.039^{*}$ \\
\hline Left rotation & $63.18 \pm 13.04$ & $57.78 \pm 11.74$ & $0.039^{*}$ \\
\hline Copenhagen neck function disability index & $7.27 \pm 3.91$ & $9.76 \pm 4.34$ & $0.004^{*}$ \\
\hline
\end{tabular}

Values present as mean \pm SD were analyzed by independent samples t-test. *: Significant.

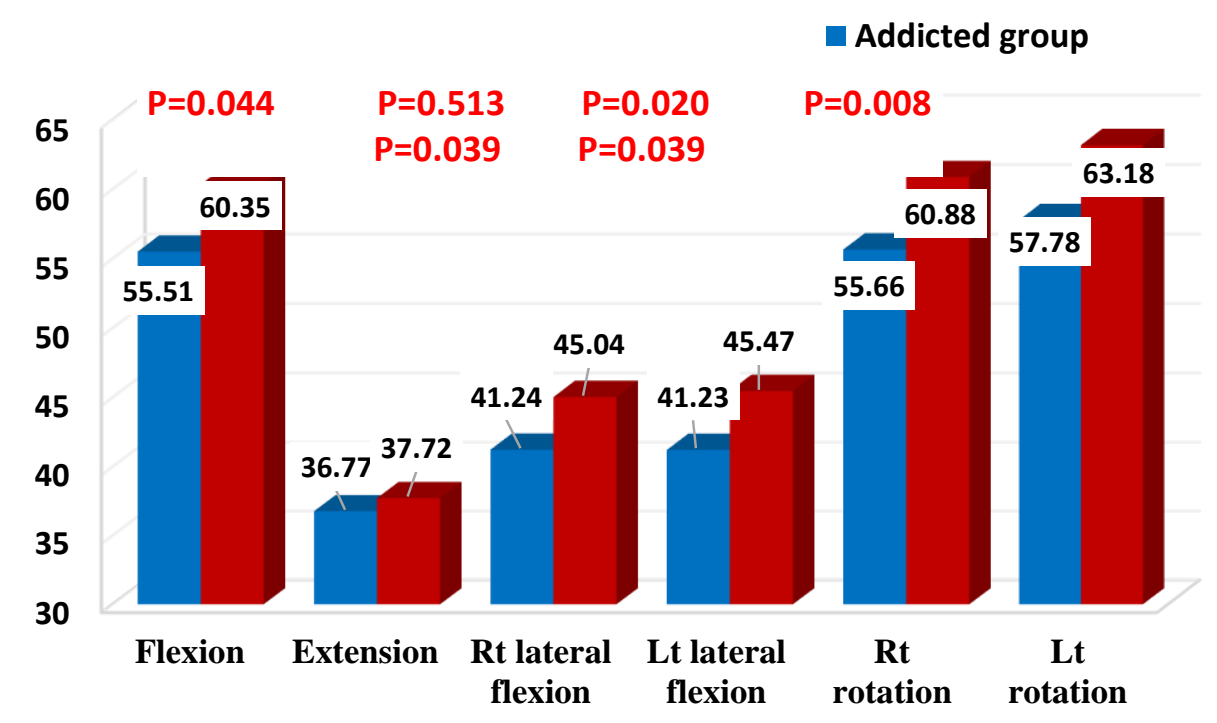

Figure (1): Range of motion among both groups. 
ejhm.journals.ekb.eg

\section{DISCUSSION}

This study was conducted to examine the effect of smartphone addiction on the, cervical ROM (flexion, extension, left lateral flexion, right lateral flexion, left rotation and right rotation), and cervical functions among undergraduate physical therapist.

With regard to smartphone addiction scale short version, the mean smartphone addiction scale sv was statically significantly higher among smartphone addicted group $(\mathrm{P}<0.001)$.

With regard to the mean Copenhagen neck functional disability index, it was significantly higher among smartphone addicted group $(\mathrm{P}=0.004)$, nearly half of smartphone addicted group (52.6\%) were functionally affected regarding CNFDI (score 10-20) compared to $29.0 \%$ of normal group with statistically significant difference $(\mathrm{P}=0.021)$.

The study of Shah et $\boldsymbol{a l} .{ }^{(16)}$, examined the effect of smartphone addiction and relation to musculoskeletal disorder specially text neck by SAS questionnaire into English language and neck disability index (NDI) by 100 physical therapy students in Ahmebad College, SBB College and VS Hospital their age $20-25$ years old found that positive correlation between the smartphone addiction and neck disability with predominance of female seen that may later effect on long term disability

A cross sectional study applied over 2435 participants in Saudi Arabia by (sharan,.2015) ${ }^{(17)}$, their ages ranged from $14-18$ years old, using online google survey form, which was distributed through social media, found out positive correlation between neck pain, dysfunction and long term smart devices usage.

The physical exposure while text messaging on a mobile phone consists of low physical load, and excessive neck flexion. If text neck is left untreated, then it can lead to some serious permanent damage, such as flattening of the spinal curve, early arthritis; spinal misalignment, spinal degeneration or disc compression, disc herniation, or nerve damage, occupational overuse syndrome or repeated stress/strain injury ${ }^{(\mathbf{1 8})}$. This illustrates the result of this study that the smartphone addiction lead to long term neck disability.

The smartphone overuse increases solicitation and repeated movement of tendons and joints. This is exaggerated by holding mobile for long time. Repeated typing and calling finally affect muscle activity and effort. There is also increase in the count of neutrophils during calls of smartphone leading to pro inflammation stimuli on musculoskeletal systems which develop fatigue and disability ${ }^{(19)}$. Extension isn't a functional ROM so smartphone addiction wouldn't affect it for this reason.

Study of Collier ${ }^{(20)}$ found that long and continues usage of visual terminal devises causes incorrect posture as hunched posture, neck flexion posture and damage surrounding soft tissues and ligaments at spine. The main cause of decrease mobility of cervical spine is cervical pain even in short term and disability.

Gustafsson_et al. (8) had a longitudinal population-based cohort study with Swedish young adults aged 20-24 years. Data were collected via a web-based questionnaire at baseline $(\mathrm{n}=7092)$ and after five years, the results revealed long-term effects on musculoskeletal disorders at neck and its function. This agreed with the study results as smartphone addiction for at least 3 years would lead to neck disability.

Lunge and Kokiwar ${ }^{(21)}$ had assessed a cross sectional Indian study upon 306 medical students; their ages ranged from $21-23$ years old. The medical students applied self-reporting questionnaire. The result revealed that using of gadgets takes a prevalent part in the daily lives of medical students and would affect the daily life activities. This agreed with the current study at the activity of daily life around neck region.

Inal et $\boldsymbol{a l} .{ }^{(22)}$ had a Turkish study done upon 319 university students by using SAS questionnaire. The findings revealed that daytime dysfunction scores were higher in the high smartphone-use group than in the low smartphone-use group. This result agreed with the current study.

A cross-sectional study was conducted by Alosaimi et al..$^{(23)}$ on 2367 university students in King Saud University. Electronic self-administered questionnaire and the problematic use of mobile phones (PUMP) Scale were used. As a consequence of using the smartphones for 8 hours per day the students had experienced a lack of energy at the next day, this support the result of current study.

Abdulwahab et al. (24) study had a crosssectional design applied among 78 King Saud University students, to measure self- reported addiction to smartphone use and any abnormal symptoms of neck function using the smartphone addiction scale (SAS) and neck disability index (NDI) respectively. The study found a positive correlation between addiction to smartphone use and various degrees of neck problems among the participants. The Spearman correlation coefficient showed a significant correlation $(\mathrm{p}<$ 0.05 ) between SAS and NDI, this strongly agreed with the current study.

Kee et al. $^{(15)}$ conducted a study upon 100 participant recruited to Oral Medicine Department, Kyungpook National University Hospital with temporomandibular joint (TMJ) disorder assessed the smartphone addiction by SAS SV and cervical mobility by CROM. They found that there is positive correlation between smartphone addiction and limitation of cervical mobility flexion, extension, LT rotation and RT rotation. This agreed with the present study as the significant differences found between normal and addicted group but disagreed in LT and RT side flexion as the Kee's study found that there wasn't any significant differences between the 2 groups. The point of disagreement because the sample of the study had TMJ disorder. 
ejhm.journals.ekb.eg

A study of So and Woo ${ }^{(25)}$ assessed the effect of short term usage smartphone for $20 \mathrm{~min}$ and effect of smart usage upon neck pain which was measured by CROM. They found that there was a statically significant differences in flexion in both groups but if there is pain the significant differences in flexion, extension and left side flexion, this study have same result of the present study in flexion ROM but a disagreement point was because the pain taken as a factor in the assessment of ROM.

It was concluded that smartphone addiction affect cervical range of motion and neck function so, it is recommended that physical therapy students should not use smartphone in addicted manner to prevent long term neck disability and ergonomics advises for smartphone use will be one of physical therapy preventive therapy programmers.

\section{Limitation}

Participants found difficulty in understanding the procedures.

\section{Conflict of interest}

There wasn't any conflict of interest.

\section{REFERENCES}

1. Egyptian ministry of Communications and Information(2019) : Technology statistical profile MCIT. www.mcit.gov.eg/

2. Ming Z, Pietikainen S, Hänninen O (2006): Excessive texting in pathophysiology of first carpometacarpal joint arthritis. Pathophysiology J., 13(4): 269-270.

3. Yoo W and Kim M (2010): Effect of different seat support characteristic on the neck and trunk muscles and forward head postural of visual display terminal workers, Applied ergonomics J., 36:3-8.

4. Alshahrani A, Aly S, Abdrabo M and Asiri F (2018): Impact of smartphone usage cervical proprioception and balance in healthy adults. Biomedical Research J., 29(12):2547- 2552.

5. Gold J, Driban J, Thomas N, Chakran T, Channell V and Komaroff E (2012): Postures, typing strategies, and gender differences in mobile device usage: An observational study. Applied ergonomics J., 43(2): 408-412.

6. Gupt V, Arora S and Gupta M (2013):Computer-related illnesses and Facebook syndrome: what are they and how do we tackle them. Medicine Update J., 23:676-679.

7. Park J, Kim H, Kim j, Kim K, Kim N, Choi I et al. (2015) : The effects of heavy smartphone use on the cervical angle, pain threshold of neck muscles and depression. Advanced Science and Technology Letters J., 91: 12-17.

8. Gustafsson E, Johnson P and Hagberg M (2010) : Thumb postures and physical loads during mobile phone use-A comparison of young adults with and without musculoskeletal symptoms. Electromyography and Kinesiology J., 20(1):127135.

9. Berolo S and Wells A (2010) : Musculoskeletal symptoms among mobile hand-held device users and their relationship to device use: a preliminary study in a Canadian university population. Applied genomics J., 42(2): 371-378.
10. Yong M, Lee $H$ and Lee $M$ (2016) : Correlation between head posture and proprioceptive function in the cervical region. physical therapy science J., 28(3):857-860.

11. Misterska E, Jankowski R, Glowacki M (2011): Cross cultural adaptation of the Neck Disability Index and Copenhagen Neck Functional Disability Scale for patients with neck pain due to degenerative and discopathic disorders: psychometric properties of the Polish versions. BMC Musculoskelet Disord J., 12:84.

12. Lee J and Seo K (2014) : The comparison of cervical repositioning errors according to smartphone addiction grades. Physical therapy science J., 26(4):595-598.

13. Kwon M, Kim D, Cho H, Yang S(2013) : The smartphone addiction scale: development and validation of a short version for adolescents. PloS one,31: 8(12).

14. Ferreira M, Borges B, Rezende I et al. (2010) : Are neck pain scales and questionnaires compatible with the international classification of functioning, disability and health? A systematic review. Disability Rehabilitation J., 32:1539-1546.

15. Kee I, Byun J, Jung J and Choi J (2016) : The presence of altered craniocervical posture and mobility in smartphoneaddicted teenagers with temporomandibular disorders. physical therapy science J., 28(2): 339-346.

16. Shah P, Sheth $M$ (2018) : Correlation of smart phone use addiction with text neck syndrome and SMS thumb in physiotherapy students. Int J Community Med Public Health, 28(5):2512-2516.

17. Sharan D (2015): Clinical features of musculoskeletal disorders due to hand-held devices. https://www.iea.cc/congress/2015/1901.pdf

18. Neupane S, Ali U and Mathew A (2017) : Text Neck Syndrome-Systematic. Imperial Interdisciplinary Researches J., 3(7): 141-158.

19. Megna M, Gisonni P, Napolitano M, Orabona G, Patruno C, Ayala F and Balato N (2018) : The effect of smartphone addiction on hand joints in psoriatic patients: an ultrasoundbased study. the European Academy of Dermatology and Venereology J., 32(1):73-78.

20. Collier $\mathbf{R}(\mathbf{2 0 1 6 )}$ : Mental health in the smartphone era. CMAJ.,188(16):1141-1142.

21. Lunge $V$ and Kokiwar $P$ (2019): Prevalence and purposes of gadget use among medical students. International Journal Of Community Medicine And Public Health, 6(2): 500-503.,

22. Inal $E$, Demirci $K$, Çetİntürk A, Akgönül $M$, Savaş $S$ (2015): Effects of smartphone overuse on hand function, pinch strength, and the median nerve. Muscle \& nerve J., 52(2):183188.

23. Alosaimi F, Alyahya H, Alshahwan $\mathrm{H}, \mathrm{Al}$ Mahyijari $\mathrm{N}$ and Shaik S (2016): Smartphone addiction among university students in Riyadh, Saudi Arabia. Saudi medical J., 37(6):675 676.

24. AlAbdulwahab S, Kachanathu S, AlMotairi M (2017): Smartphone use addiction can cause neck disability. Musculoskeletal care J., 15(1): 10-12.

25. So Y and Woo Y (2014): Effects of smartphone use on muscle fatigue and pain and, range of motion among subjects with and without neck muscle pain. Physical Therapy cervical Korea J., 21(3): 28-37. 